\title{
Guest Editorial Sensorimotor Contingencies for Cognitive Robotics
}

\begin{abstract}
The sensorimotor approach to cognition states, that 2 the key to bring semantics to the world of a robot, requires 3 making the robot learn the relation between the actions that the 4 robot performs and the change it experiences in its sensed data 5 because of those actions. Those relations are called sensorimotor 6 contingencies (SMCs). This special issue presents a variety of 7 recent developments in SMCs with a particular focus on cognitive 8 robotics applications.
\end{abstract}

9 Index Terms-Cognitive architectures, cognitive robotics, 10 multimodal perception, sensorimotor contingencies (SMCs).

\section{Scope of This Special Issue}

${ }_{11}^{12} \mathbf{T}$ HE SENSORIMOTOR approach to cognition breaks completely the classic sense-plan-act pipe that rules most 14 of today's autonomous robots, by mixing sensation with 15 action, aiming to bridge the gap between symbolic data and 16 semantics for robots 1). Sensorimotor contingencies (SMCs) 17 are defined as the relations learned by the robot between the 18 actions that the robot performs and the change it experiences 19 in its sensed data because of those actions. The goal of bring20 ing SMCs to robotics is to build robots with a more robust 21 behavior in real environments 2), 3).

22 In order to have robots outside of controlled environment, 23 we need robots that understand their own environments 4), and 24 it looks like a way to achieve this is by making the robot build 25 by itself the SMCs that are common to every environment. ${ }_{26}$ Having robots that can generate such laws, will allow them to 27 understand the world they are immersed, and make them by 28 hence, more robust in real environments 5).

29 We selected these papers for this special issue to provide a so broad view of the subject, and particularly promoted papers 31 with practical embodied implementations that raise the current 32 challenges in the field.

3з Among all the submissions what we observe is the agree34 ment in considering the importance of multi-modal perception. 35 The papers in this Special Issue use different combinations of 36 proprioceptive sensors with visual cues and tactile sensors. We 37 note that three of them rely on tactile information.

38 Lot of efforts have been made to understand the mech39 anism of perception, obviously linked to robot actions 6). ${ }_{40}$ Unfortunately, we realize that there is a very basic use of 41 the robot capabilities, and the explored actions are still very 42 simple. We envisage that the community will start soon to 43 use more complex actions and take full advantage of the great 44 robotic platforms we currently have available in research.

Digital Object Identifier 10.1109/TCDS.2017.2703758

\section{Contribution to the Special Issue}

The special issue is composed of five papers. The most the- 46 oretical of the selected works is presented by Lanillos et al. ${ }_{47}$ in the paper entitled Yielding Self-Perception in Robots 48 Through Sensorimotor Contingencies, where authors address 49 the problem of self-perception, including a novel model for 50 self-detection, which does not need to track or store the body 51 parts. Self-perception, taken as the understanding of the sen- 52 sory consequences of performing an action, can improve the 53 capabilities of the robot to interact in unknown environments. 54 They propose that the concept of usability can be emerged 55 this way. As perception they use artificial skin and visual cues, 56 and the output is the discovery of the potential usable objects. 57 For that purpose, an experiment is designed for discovering 58 usable objects in the scene based on the taping or pushing 59 setup proposed lately in [item 7) in the Appendix].

A different approach of this same idea, along with its imple- 61 mentation in a real robot, is presented by Zambelli and Demiris 62 in their paper Online Multimodal Ensemble Learning Using 63 Self-Learnt Sensorimotor Contingencies. Authors introduce a 64 learning architecture where knowledge emerges from robot 65 interactions and multimodal sensory system. The method pro- 66 duces predictors able to relate motor commands to perceptions ${ }_{67}$ of the robot sensory system. Notably, it does not require prior 68 information about the kinematic or dynamic models of the 69 robot. Hence, the proposed framework, initially developed 70 using an iCub humanoid robot 8) and a piano keyboard, can 71 be applied to different robotic platforms.

The manuscript by Giakos et al. entitled Perception of ${ }_{73}$ Object Features During Robotic Sensorimotor Development 74 tackles the problem of perception, and proposes a mechanism 75 to think in a stable representation of the world inspired in 76 infant learning. The system is based on observing how the 77 gaze control impacts on the perception of the objects, and 78 provide an interesting insight on the emergence of features 79 that can lead to object recognition. Experiments give a full 80 demonstration of a longitudinal development of both senso- 81 rimotor development and early object perception on an iCub 82 humanoid robot.

The paper by Marcel et al. entitled Building a Sensorimotor 84 Representation of a Naive Agent's Tactile Space tackles the 85 problem of the discovery of the inherent structure of the 86 interaction with the robot body. This work is focused on 87 the extension of the approach in [item 9) in the Appendix] 88 to the building of an internal representation of an agent body, 89 in the vein of Frolov's previous work. Authors present a 90 method to build a perception of the environment, and provide 91 a formalization of the so-called "sensorimotor invariants." The 92 method is shown to be valid also to consider motion planning. 93 
$94 \quad$ The work by Arriola-Rios and Wyatt entitled A Multi-Modal 95 Model for Prediction and Classification of Object Deformation ${ }_{96}$ During Robotic Manipulation introduces a method to learn 97 deformation models from robot interaction. Manipulation of 98 3-D deformable objects is a challenging task 10); in this paper 99 authors propose a method to predict the forces and behavior 100 after the contact, and at the same time classify the material. ${ }_{101}$ Compared to other works with simple use-cases, here authors ${ }_{102}$ can deal this challenging task because some knowledge is ${ }_{103}$ parameterized in the system, and the SMC is used to learn 104 some of the parameters, demonstrating that SMC approaches 105 are useful also as partial learners.

106 Guillem Alenya, Lead Guest Editor

107 Institut de Robòtica i Informàtica Industrial CSIC-UPC

108 Perception and Manipulation Group

10908028 Barcelona, Spain

110 e-mail: galenya@iri.upc.edu

111 Ricardo Tellez, Guest Editor

112 Institut de Robòtica i Informàtica Industrial CSIC-UPC

${ }_{113}$ Perception and Manipulation Group

${ }_{114} 08028$ Barcelona, Spain

115 e-mail: rtellez@iri.upc.edu

116 KeVin O’Regan, Guest Editor

117 Institut Paris Descartes de Neurosciences et Cognition CNRS

118 Laboratoire Psychologie de la Perception

11975270 Paris, France

120 e-mail: jkevin.oregan@gmail.com

121 Cecilio Angulo, Associate Editor

122 Universitat Politècnica de Catalunya

123 Automatic Control Department

${ }_{124} 08028$ Barcelona, Spain

125 e-mail: cecilio.angulo@upc.edu

\section{APPENDIX \\ RELATED WORK}

1) J. K. O'Regan and A. Noë, “A sensorimotor account of vision and 128 visual consciousness," Behav. Brain Sci., vol. 24, no. 5, pp. 939-973, 129 2001.

2) A. Maye and A. K. Engel, "A discrete computational model of sen- 131 sorimotor contingencies for object perception and control of behavior," 132 in Proc. IEEE Int. Conf. Robot. Autom., Shanghai, China, May 2011, 133 pp. 3810-3815.

3) A. Maye and A. K. Engel, "Extending sensorimotor contingency 135 theory: Prediction, planning, and action generation," Adapt. Behav. 136 Animals Animats Softw. Agents Robots Adapt. Syst., vol. 21, 137 no. 6, pp. 423-436, Dec. 2013. [Online]. Available: http://dx.doi.org/ 138 $10.1177 / 1059712313497975$

4) C. Angulo, R. A. Téllez, and D. E. Pardo, "Internal representa- 140 tion of the environment in cognitive robotics," Int. J. Robot. Autom., 141 vol. 24, no. 3, 2009. [Online]. Available: http://www.actapress.com/ 142 Abstract.aspx? paperId $=35377$

5) T. Buhrmann, E. Di Paolo, and X. Barandiaran, "A dynamical 144 systems account of sensorimotor contingencies," Front. Psychol., vol. 4, 145 p. 285, May 2013. [Online]. Available: http://journal.frontiersin.org/ 146 article/10.3389/fpsyg.2013.00285

6) A. K. Seth, "A predictive processing theory of sensorimo- 148 tor contingencies: Explaining the puzzle of perceptual presence 149 and its absence in synesthesia," Cogn. Neurosci., vol. 5, no. 2, 150 pp. 97-118, 2014. [Online]. Available: http://www.biomedsearch.com/ 151 nih/predictive-processing-theory-sensorimotor-contingencies/24446823. 152 html

7) R. Saegusa, G. Metta, G. Sandini, and L. Natale, "Developmental per- 154 ception of the self and action," IEEE Trans. Neural Netw. Learn. Syst., 155 vol. 25, no. 1, pp. 183-202, Jan. 2014.

8) G. Metta et al., "The iCub humanoid robot: An open-systems platform 157 for research in cognitive development," Neural Netw., vol. 23, nos. 8-9, 158 pp. 1125-1134, 2010. [Online]. Available: http://www.sciencedirect.com/ 159 science/article/pii/S0893608010001619

9) A. Laflaquière, J. K. O'Regan, S. Argentieri, B. Gas, and A. V. Terekhov, 161 "Learning agent's spatial configuration from sensorimotor invariants," 162 Robot. Auton. Syst., vol. 71, pp. 49-59, Sep. 2015. [Online]. Available: 163 http://dx.doi.org/10.1016/j.robot.2015.01.003

10) F. Khalil and P. Payeur, Dexterous Robotic Manipulation 165 of Deformable Objects With Multi-Sensory Feedback-A 166 Review. INTECH Open Access, 2010, pp. 587-619. [Online]. 167 Available: https://www.intechopen.com/books/robot-manipulators- 168 trends-and-development

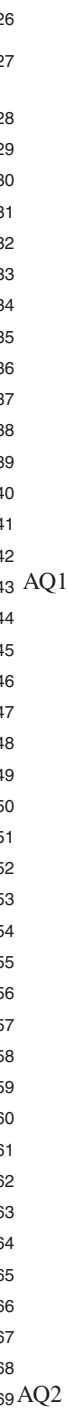

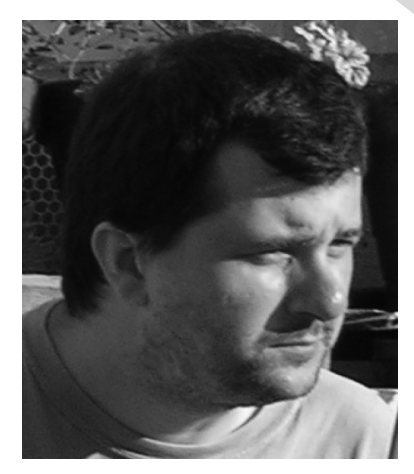

Guillem Alenya received the Ph.D. (Doctor Europeus) degree from the Polytechnic University 170 of Catalonia, Barcelona, Spain, in 2007, with a research on mobile robot navigation using active 171 contours.

He holds a tenured position with Spanish Scientific Research Council CSIC, Madrid, Spain. 173 His Ph.D. research was partly developed with the Robosoft company in France, where he was 174 supported by a EU-FP6 Marie-Curie scholarship. He has been a visitor with Karlsruhe Institute of 175 Technology, Karlsruhe, Germany, in 2007, INRIA, Grenoble, France, in 2008, and Bristol Robotics 176 Laboratory, Bristol, U.K., in 2016. He has participated in numerous scientific projects involving 177 image understanding, next-best-view, rule learning, and plan execution tasks. He is currently a 178 local IP of the EU MSCA training network SOCRATES, a co-IP of the EU project IMAGINE, 179 and a Leader of numerous technological transfer projects. From 2009 to 2011, he served as the 180 Head of the Robotics Department. 


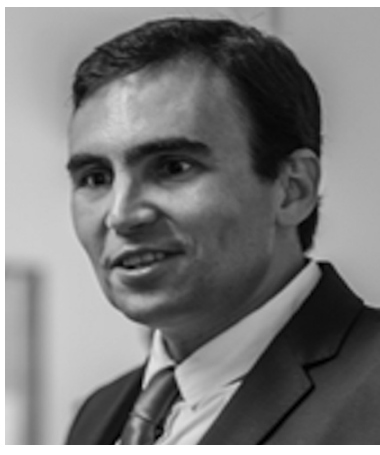

Ricardo Tellez received the Ph.D. degree in artificial intelligence from Universitat Politècnica 182 de Catalunya, Barcelona, Spain, with a research about the generation of dynamic gaits in real 183 quadruped robots using evolutionary algorithms.

He is currently a CEO and a Founder of the Construct, a robotics software company. He was 185 a Post-Doctoral Fellow with Spanish Scientific Research Council CSIC, Madrid, Spain. He has 186 over 10 years experience in the service robots industry, specially researching in the development 187 of humanoid robots at PAL Robotics. He has served as a Team Leader several times in service 188 robots competitions integrating industry and academia partners. He has been also participating in 189 numerous technological transfer projects.

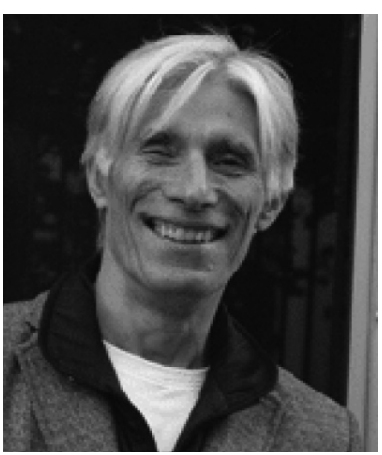

Kevin O'Regan is an Ex-Director of the Laboratoire Psychologie de la Perception, CNRS, 192 Universite Paris Descartes, Paris, France. After early research on eye movements in Reading, he 193 was led to question established notions of the nature of visual perception, and to discover, with 194 collaborators, the phenomenon of "change blindness." He has published a book entitled Why Red 195 Doesn't Sound Like a Bell: Understanding the Feel of Consciousness (Oxford University Press) in 196 2011. In 2013, he received a five year Advanced ERC grant to explore his "sensorimotor" approach 197 to consciousness in relation to sensory substitution, pain, color, space perception, developmental 198 psychology, and robotics.

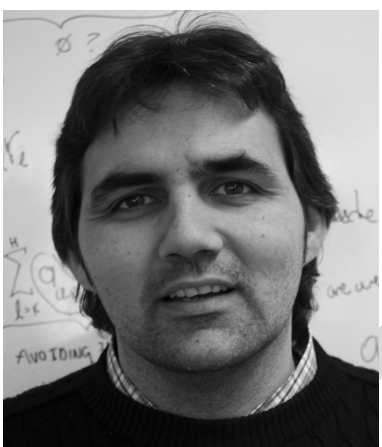

Cecilio Angulo received the B.Sc. and M.Sc. degrees in mathematics from the University of 201 Barcelona, Barcelona, Spain, in 1993, and the Ph.D. degree in sciences from the Department of 202 Automatic Control, Technical University of Catalonia (UPC), Barcelona, in 2001.

He was an Assistant Professor with the Department of Informatics and Applied Mathematics, 204 University of Girona, Girona, Spain. He also carried out a Pre-Doctoral Fellow with LP2A, 205 Universite de Perpignan, Perpignan, in 1999 with Dr. M. Polit, a Post-Doctoral Fellow with 206 IIIA, CSIC, Catalonia, Spain, in 2004 with Dr. P. Garcia, and a Post-Doctoral Fellow with DIBE 207 Univeristà degli Studi di Genova, Genoa, Italy, in 2006 with Dr. D. Anguita. He became an 208 Associate Professor with the Department of Automatic Control in 2007. He served as the Director 209 of the master's degree in Automatic Control and Robotics, UPC, for five years. His current 210 research interests include machine learning, human-robot interaction, cognitive robotics, and body 211 sensors.

Dr. Angulo is an Associate Editor of the IEEE TRAns ACTIOns ON CoGNITIVE AND DEVELOPMENTAL SySTEMS, and has 213 also served as the Chair for several international conferences. 


\section{AUTHOR QUERIES \\ AUTHOR PLEASE ANSWER ALL QUERIES}

PLEASE NOTE: We cannot accept new source files as corrections for your paper. If possible, please annotate the PDF proof we have sent you with your corrections and upload it via the Author Gateway. Alternatively, you may send us your corrections in list format. You may also upload revised graphics via the Author Gateway.

AQ1: Please provide the page range for Reference [4].

AQ2: Please provide the publisher location for Reference [10].

AQ3: Please provide the educational background for the author "K. O'Regan." 